\title{
A Pulse Signal Generator for the Testing of Neutron Pulse Counters
}

\author{
Chuanxi Jin, Lifeng Guo \& Panhui Chen \\ Naval University of Engineering, Wuhan 430033, China
}

ABSTRACT: A pulse signal generator is designed to deal with the testing of the pulse counters for the measurement of the neutron fluence rates. It contains a microcontroller and a pulse generator circuit which consist of a high speed DA converter and a clock based on DDS to realize a square wave output with a variable frequency of $0 \sim 2 \mathrm{MHz}$ and a variable amplitude of 0 20VP-P. The test shows that it can work stability with a high precision frequency.

KEYWORD: pulse counters; DA converter; DDS

\section{INTRODUCTION}

In the start-up process of the nuclear reactors, the neutron fluence rates measurement can be used to monitor the state of the reactor and it is very important to the safety of the reactor. As the value of neutron fluence rate varies from $10^{-2}$ to $10^{10} \mathrm{n} / \mathrm{cm}^{2} \mathrm{~s}$, the measurement usually using the method of segmentation (RF Fan et al, 2004). Counters are used to record the pulse output from the neutron counter tubes to calculate the neutron fluence rates when the value of neutron fluence rates below $10^{3} \mathrm{n} / \mathrm{cm}^{2} \mathrm{~s}(\mathrm{CY}$ Liu et al, 2012) (Marcus Fedrizzi et al, 2015). A neutron ionization chamber used to transform the neutron fluence rates to a micro current output.

In order to test the circuit of the pulse counter, a pulse signal generator with a variable frequent and amplitude is designed in this paper ( $\mathrm{Y} \mathrm{Wu}, 2011)$
(Hsinchuan Chen, 2008). According to the performance parameter of the pulse counter, the frequency of the pulse signal generator ranges from $0 \mathrm{MHz}$ to 2.000MHz with a $1 \mathrm{KHz}$ step and the amplitude ranges from $0 V_{P-P}$ to $20 V_{P-P}$.

\section{CIRCUIT DESIGN}

The pulse signal generator mainly consists of a microcontroller, a programmable clock, a pulse generator circuit and an output driver circuit. The microcontroller used to accept the set parameter of the user interface and control the programmable clock and a pulse generator circuit. The Schematic diagram of the pulse signal generator is described in figure 1 .

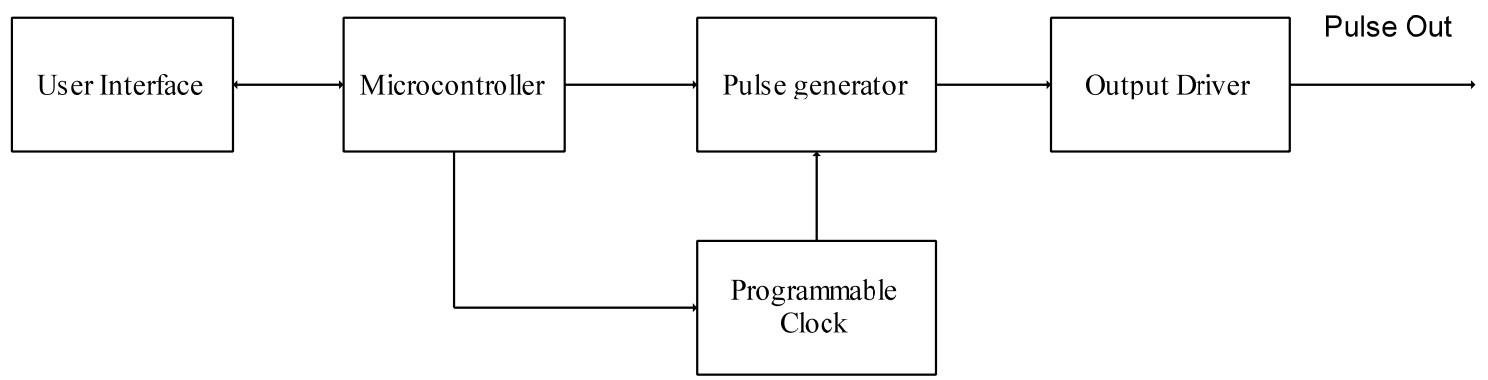

Figure 1 Schematic diagram of the pulse signal generator

In this paper, we define the square wave as the output waveform, and a DA converter DAC908 is used to produce a square wave. As the DAC908 set the output voltage at the rising edge of the clock, a square wave can be produced when the input code of DAC908 changed between 0 to code D which related to the amplitude at the two adjacent rising edges. According to the principle of square wave genera- 
tion, the DA converter needs to complete two times conversation in every period. Because of the maximum frequency of the output are $2 \mathrm{MHz}$ and the period of the $2 \mathrm{MHz}$ square wave is $250 \mathrm{~ns}$, the DAC908 with the setting time of 30ns meet the requirement. Therefore the clock range of DAC is
$0 \sim 4.000 \mathrm{MHz}$ which is equal two times of the output frequency.

In order to achieve the synchronous switching of the input code of DA converter, a counter circuit $74 \mathrm{HC} 393$ and a latch circuit $74 \mathrm{HC} 573$ are used to deal with the sequential logic which is described in figure 2 .

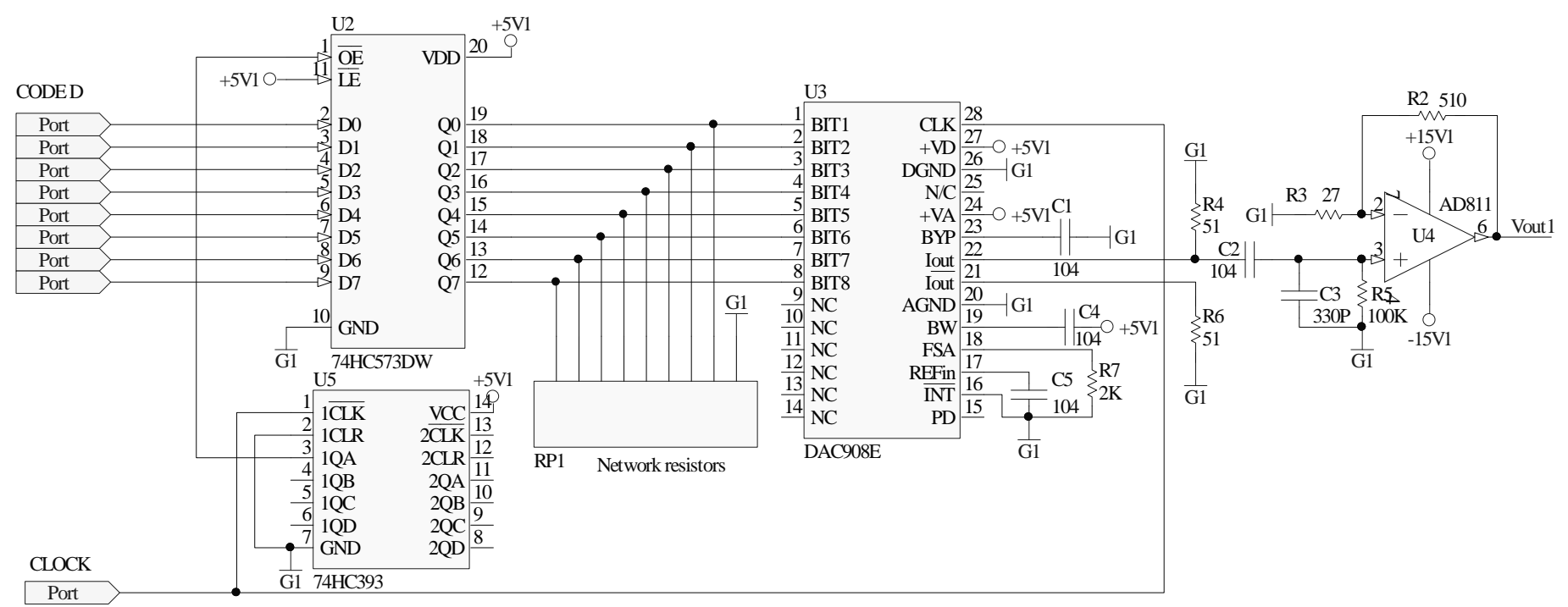

Figure 2 Circuit of the pulse generator

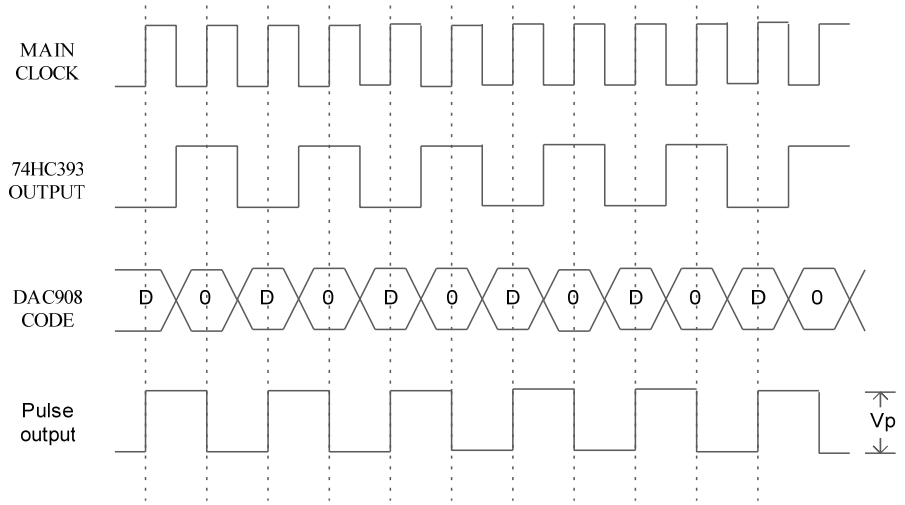

Figure 3 Sequential logic diagram
The output of 74HC393 divides the main clock to 0.5 times and connected to the output enable pin of $74 \mathrm{HC} 573$. When the $74 \mathrm{HC} 393$ output is " 0 ", the code D will be transmitted by $74 \mathrm{HC} 573$ to the input of DAC908.Otherwise, the output of 74HC573 will be pulled to " 0 " levels by the resistor network when the $74 \mathrm{HC} 393$ output is " 1 ". As the rising edge of the main clock comes to the middle time of every " 0 " or " 1 " period of $74 \mathrm{HC} 393$ output, the output of DAC908 alternate between 0 to Vp which corresponded to code $\mathrm{D}$ at every to period of the main clock. The sequential logic diagram is described in figure3.

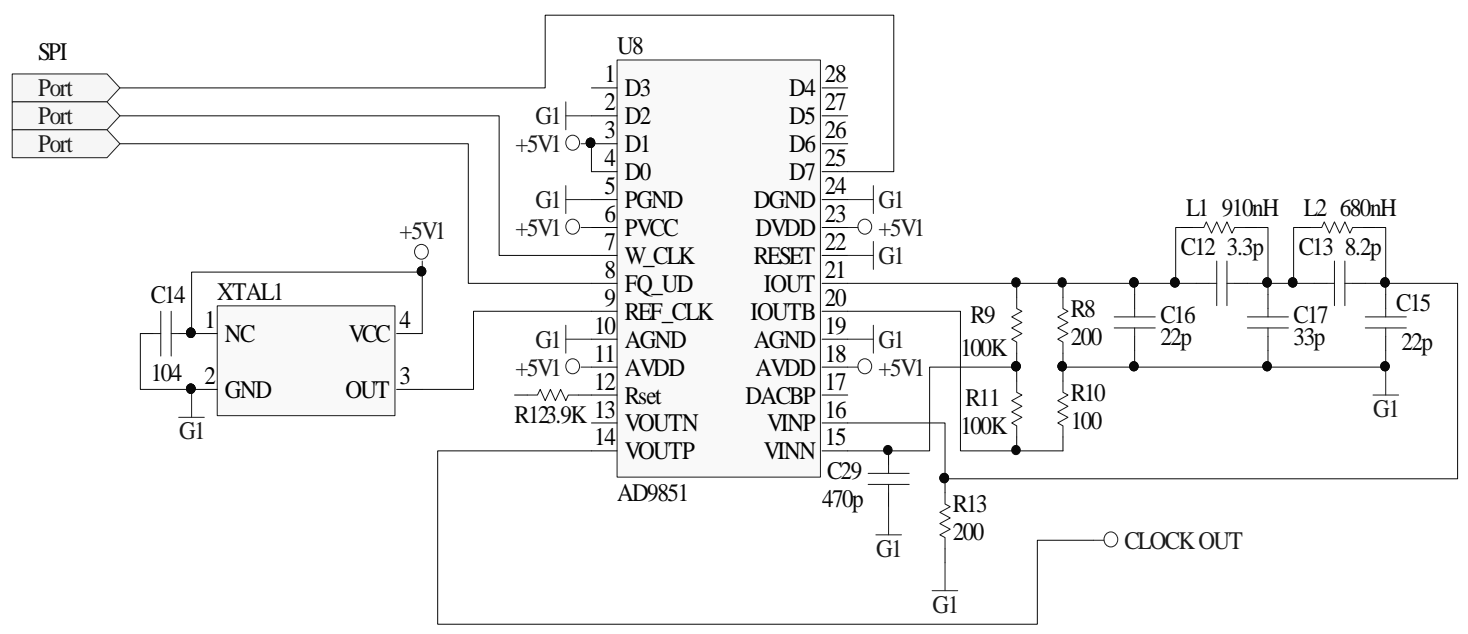

Figure 4 The programmable clock circuit 
A DDS chip AD9851 is used to generate the main clock ranges from $0 \mathrm{~Hz}$ to $4 \mathrm{MHz}$ with a step of 2 KHz. An active crystal of $100 \mathrm{MHz}$ is used to be the reference clock of the AD9851, and the interface which connected to the microcontroller is set at SPI mode to simplify the wire connection. In order to improve the stabilization of the clock output, a passive filter is designed to reduce the phase noise of the sine wave output and the inner comparator is used to reshaping the sine wave to square wave. The programmable clock circuit is shown in figure 4.

\section{ALGORITHM DESIGN}

The software design of pulse signal generator is mainly about the code for the microcontroller. It mainly contains the calculate method of the frequency tuning word and the system process controlling.

The PLL circuit in AD9851 is a 6 times multiplier to raise the system clock with the power dissipation raised up. In order to reduce the power dissipation, a $100 \mathrm{MHz}$ active crystal acts as the system clock with inner PLL circuit closed.

The decimal value of 32-bit frequency tuning words $\triangle$ Phase are stored in four 8-bit registers. According to the principle of AD9851, the frequency of the output signal $f_{\text {out }}$ is related to the system clock $f_{\text {in }}$ and $\Delta$ Phase which can be calculated as:

$f_{\text {out }}=\left(\Delta\right.$ Phase $\left.\times f_{\text {in }}\right) / 2^{32}$

As the system clock equals $100 \mathrm{MHz}$, the fout can be further calculated as:

$$
\Delta \text { Phase }=f_{\text {out }} \times 85.899346
$$

The system process of the microcontroller mainly contains the user interface detection, the frequency tuning word calculation, the amplitude controlling and the parameter display. The flowchart of the microcontroller is shown in figure 5.

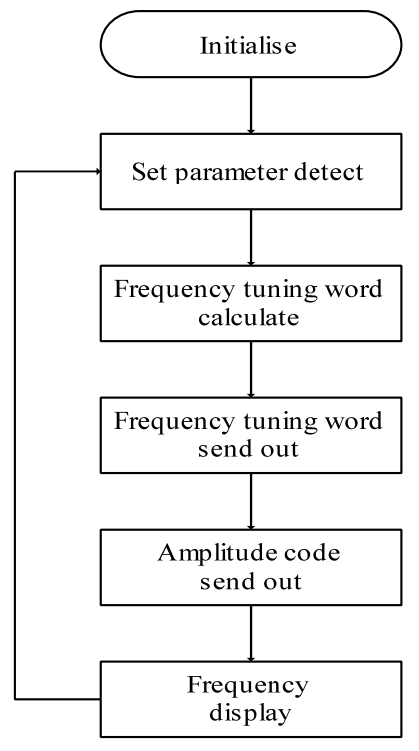

Figure 5 The flowchart of the microcontroller

\section{CIRCUIT TESTING}

A four channel pulse signal generator has been developed to confirm the pulse generator design, and a digital storage oscilloscope TDS2012B is used to test the output signal which is shown in figure 6 .

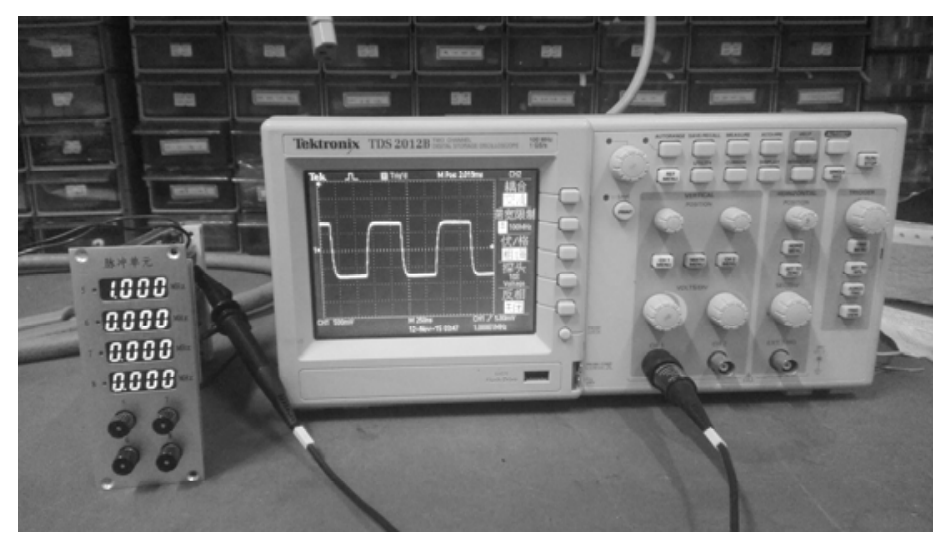

Figure 6 Circuit testing

As the frequency setting parameter varies from $0 \mathrm{MHz}$ to $2.000 \mathrm{MHz}$, the output frequency values which measured by the oscilloscope are recording in table 1 . According to the result of the frequency testing, the frequency error equals $0.01 \%$.

Table 1 Frequency testing recording

\begin{tabular}{llll}
\hline Numble & $\begin{array}{l}\text { Frequency setting } \\
(\mathrm{MHz})\end{array}$ & $\begin{array}{l}\text { Frequency measured } \\
(\mathrm{MHz})\end{array}$ & $\begin{array}{l}\text { Error } \\
(\mathrm{Hz})\end{array}$ \\
\hline 1 & 0.001 & 0.00100001 & 0.01 \\
2 & 0.010 & 0.0100001 & 0.1 \\
3 & 0.100 & 0.100001 & 1 \\
4 & 1.000 & 1.00001 & 10 \\
5 & 2.000 & 2.00002 & 20 \\
\hline
\end{tabular}




\section{CONCLUSION}

The pulse signal generator designed in this paper can generals a square wave with the amplitude and frequency variable. The circuit can work steadily with a high precision of frequency. However the pulse width is equal to half of the periods, some pulse counters need to set the pulse width to a fixed time. A pulse signal generator with the pulse width adjustable will be developed in the next step.

\section{REFERENCES}

CY Liu, LI Wen-Jie: Study on Double Range Neutron Fluence Rate Detector, Atomic Energy Science \& Technology, Vol 46(2012) No. 9, p. 1138.

Hsin-Chuan CHEN: Design of a High-Precision DDS-Based Configureable Clock Generator, IECE TRANS. ELECTRON, Vol E91-C (2008) No. 7, p. 1151.

Marcus Fedrizzi and Julio Soria: Application of a single-board computer as a low-cost pulse generator, Measurement Science and Technology, Vol 26(2015), p.1.

RF Fan, MA Jing-Fang, DX Yang: Neutron detector of highefficiency arrays of BF_3 proportional counters, Nuclear Electronics \& Detection Technology, Vol 24 (2004) No. 3, p.278.

Y Wu: DDS+PLL design of clock source based on AD9854, Electronic Measurement Technology, Vol 34 (2011) No. 8, p. 60 . 